Research Article

\title{
Hydraulic-Mechanical Coupling Model with Dual-Porosity Dual-Permeability for Anisotropy Coal Seams and Its Application in Mine Gas Extraction
}

\author{
Bingjie Huo, ${ }^{1}$ Xuedong Jing, ${ }^{1}$ Aiping $\mathrm{He}^{2}$ and Chaojun Fan $\mathbb{D}^{1,3}$ \\ ${ }^{1}$ College of Mining, Liaoning Technical University, Fuxin 123000, China \\ ${ }^{2}$ College of Safety Science and Engineering, Liaoning Technical University, Fuxin 123000, China \\ ${ }^{3}$ Energy and Mineral Engineering, G3 Center and EMS Energy Institute, Pennsylvania State University, University Park, \\ PA 16802, USA \\ Correspondence should be addressed to Chaojun Fan; chaojunfan@139.com
}

Received 12 June 2019; Revised 20 July 2019; Accepted 25 July 2019; Published 5 August 2019

Academic Editor: Robert Černý

Copyright (c) 2019 Bingjie Huo et al. This is an open access article distributed under the Creative Commons Attribution License, which permits unrestricted use, distribution, and reproduction in any medium, provided the original work is properly cited.

\begin{abstract}
The parameters of pore-fracture structure and permeability have a controlling effect on the behaviors of gas adsorption/desorption and transportation in coal reservoir. A mathematic model for coal seams is of great significance to evaluate the mass migration within the coal fracture-matrix system. In this paper, the hydraulic-mechanical coupling model considering both dual-porosity dual-permeability and anisotropy characteristics is first established by using the methods of theoretical analysis, nuclear magnetic resonance (NMR) test, and numerical simulation. Then, this model is applied to simulate the gas migration characteristics of No. 3 coal seam in Changping Mine, China. Results show that the pore structure of No. 3 coal seam is characterized by small radius of pores and pore throats, which is determined by the NMR test, verifying the dual-permeability dual-porosity of coal seams. Both matrix permeability and fracture permeability increase approximately linearly with the process of mine gas extraction. The increased magnitude of the matrix permeability is greater than that of the fracture permeability. The permeability is inversely proportional to the anisotropy coefficient. The pressure gradient within the coal matrix and fracture increases first and then decreases with the extraction time. This pressure gradient is proportional to the anisotropy coefficient at the early stage of extraction and is inversely proportional to the anisotropy coefficient at the later stage. The seepage and diffusion flux are proportional to the anisotropy coefficient. The proportion of matrix-to-fracture seepage flux to the total flux increases first and then decreases to a certain value. The proposed model provides a guide for accurate designation of gas extraction from coal seams.
\end{abstract}

\section{Introduction}

Coalbed methane (CBM), a kind of unconventional natural gas, is a clean energy source for solving the problem of global warming $[1,2]$ and also a valuable by-product of $\mathrm{CO}_{2}$ geological sequestration in coal seams [3-5]. Meanwhile, coal mine gas is one of the most serious threats to mine safety $[6,7]$. With the increase in mining depth, gas pressure and content in coal seams increase, and this imposes gas emission and serious gas dynamic disasters. Gas extraction and utilization can increase energy supply and reduce coal mine gas accidents, thus achieving remarkable social and economic benefits [8-10]. Gas migration in coal seams is the core of gas extraction, which involves the interaction of coal and gas, namely, the hydraulic-mechanical coupling responses $[11,12]$.

The pore structure of the coal seam determines the law of gas adsorption/desorption, diffusion, seepage, and mass transfer between the coal fracture and matrix $[13,14]$. The hydraulic-mechanical coupling model in the coal seam can fully reflect the relationship between gas seepage and coal deformation and capture the evolution of coal porosity and permeability [15]. For gas migration in coal seams, many gas-solid coupling models have been put forward. Zhang et al. pointed out the geostress and gas pressure control the effective stress on coal, which will change the porosity and 
permeability within the coal seam, and developed a nonlinear coupled mathematical model [16]. Considering gas desorption-induced swelling, Connell developed a coupled model for coalbed methane extraction. In this model, the permeability in the coal seam is sensitive to stress and pore pressure changes [17]. Wu et al. established a model to describe the evolution of porosity and permeability in fractured coalbeds infiltrated with gas [18]. A coupled model was presented to analyze fluid flow and deformation in porous and fractured rock considering the Klinkenberg effect [19-21]. The complex coal and gas interactions under variable temperatures were investigated [22-24]. Suites of governing equations for the hydraulic-mechanical model including the dual-porosity structure of the coal seam were developed to model the hydraulic fracturing in the coal seam and optimize the enhanced coalbed methane recovery using $\mathrm{CO}_{2} / \mathrm{N}_{2}$ mixtures [25-27]. Cao et al. developed a coal-rock solid-gas coupling mesomechanical test device, which provides a new idea for fluid-solid coupling verification [28]. To determine the pore structure of the coal seam, Yao et al. and Zhou et al. studied the pore structure of the granite by nuclear magnetic resonance (NMR) technology, which provides a basis for rock microstructure and damage mechanism $[29,30]$. Ren et al. analyzed the pore diameter and effective porosity characteristics of low-rank coal reservoirs [31]. The coal seam is characterized by a typical dualporosity system with a microporous matrix and macroporous cleats/fractures containing free and adsorbed gases $[12,13]$.

The established models are mostly isotropic single-porosity single-permeability model and seldom combine the pore structure characteristics of the coal seam with the coupling model. In this paper, we will creatively propose a hydraulic-mechanical coupling model by considering dualporosity and anisotropy characteristics of the coal seam on the basis of investigation of the pore structure of Changping coal samples. Then, the proposed model will be applied to simulate underground gas drainage and reveal the law of gas migration, as well as the influence of permeability anisotropy on gas drainage. This provides a theoretical basis for gas disaster prevention and control in gas-rich coal seam.

\section{Hydraulic-Mechanical Model for Dual- Porosity and Anisotropy Coal Seams}

2.1. Dual-Porosity Characteristics of Coal Seams and Basic Assumptions. The pore structure of No. 3 coal seam in Changping Coal Mine is studied using the NMR test. Figures 1 and 2 show the results of the NMR test. The pores of the coal reservoir were divided based on their size into micropores $(<0.1 \mu \mathrm{m})$, medium-large pores $(0.1 \sim 100 \mu \mathrm{m})$, and fractures $(>100 \mu \mathrm{m})$ [29]. In Figure 1, the curves of pore aperture distribution of coal samples show an independent bimodal shape. The difference between the two peaks is obvious. The pore structure is mainly micropores, accounting for $91 \% \sim 96 \%$ of the total pore volume.

The micropore and the $m$ medium-large pore are not connected. The micropore provides the most space for gas adsorption in the coal seam. The pore throat aperture which

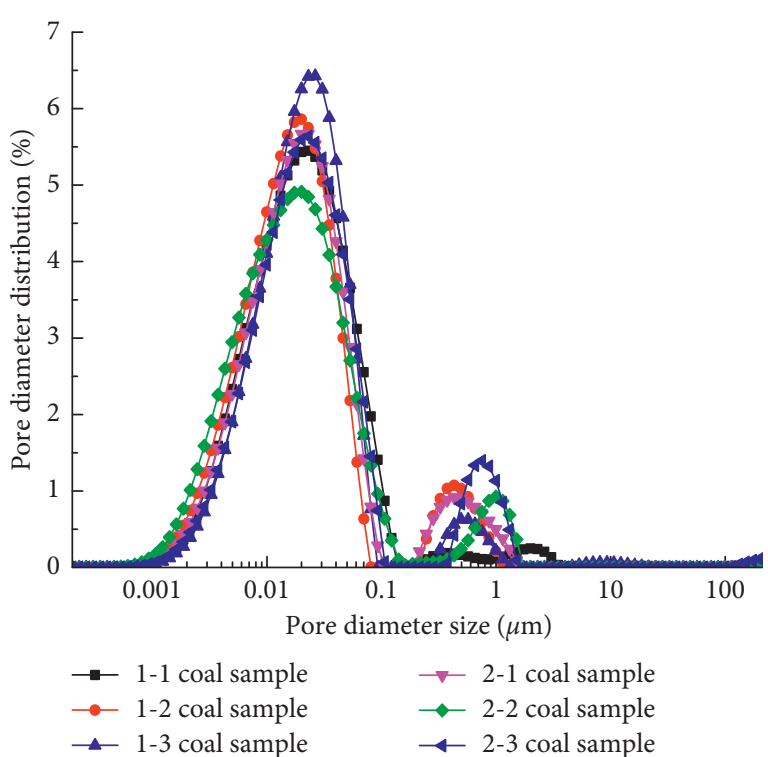

Figure 1: Pore diameter distribution in coal samples.

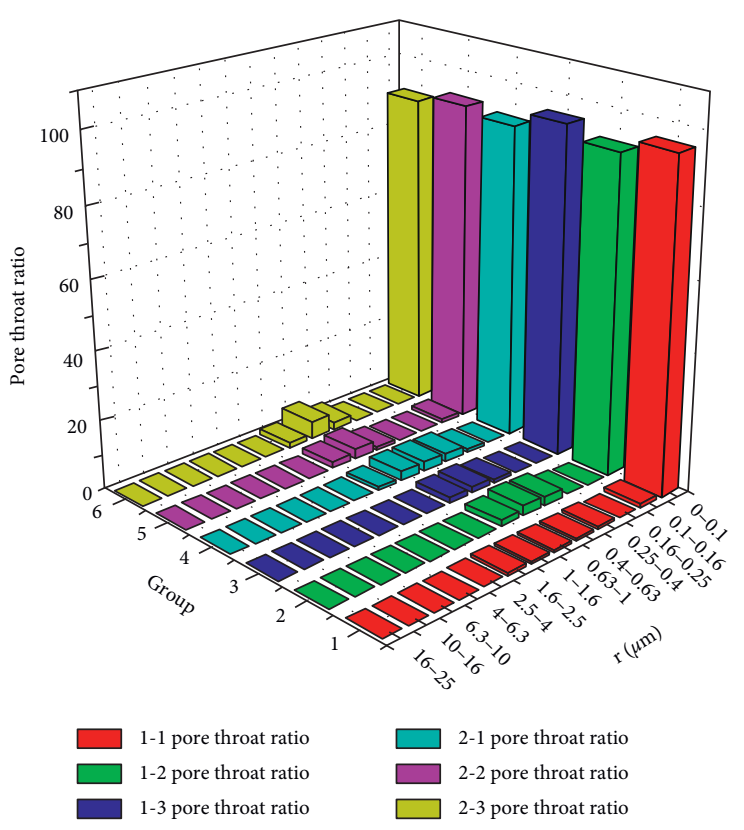

Figure 2: Distribution of pore throats in coal samples.

is smaller than $0.1 \mu \mathrm{m}$ accounts for more than $90 \%$ of all pore throats (Figure 2). A large amount of gas is stored in the micropore, which leads to the increase in the gas content in the coal seam. Hence, the dual-porosity structure conforms to the pore characteristics.

According to the pore structure characteristics, gas existence, and migration in the coal seam, the following assumptions are drawn [23, 32, 33]: (1) as shown in Figure 3, the coal seam is regarded as the anisotropic dual-porosity, dual-permeability elastic continuity medium, and the coal seam consists of fractures and coal matrix, while pores exist in the coal matrix; (2) the relationship between adsorbed gas content and gas pressure follows the Langmuir equation; (3) gas is assumed to be satisfied with ideal gas; (4) the coal seam 


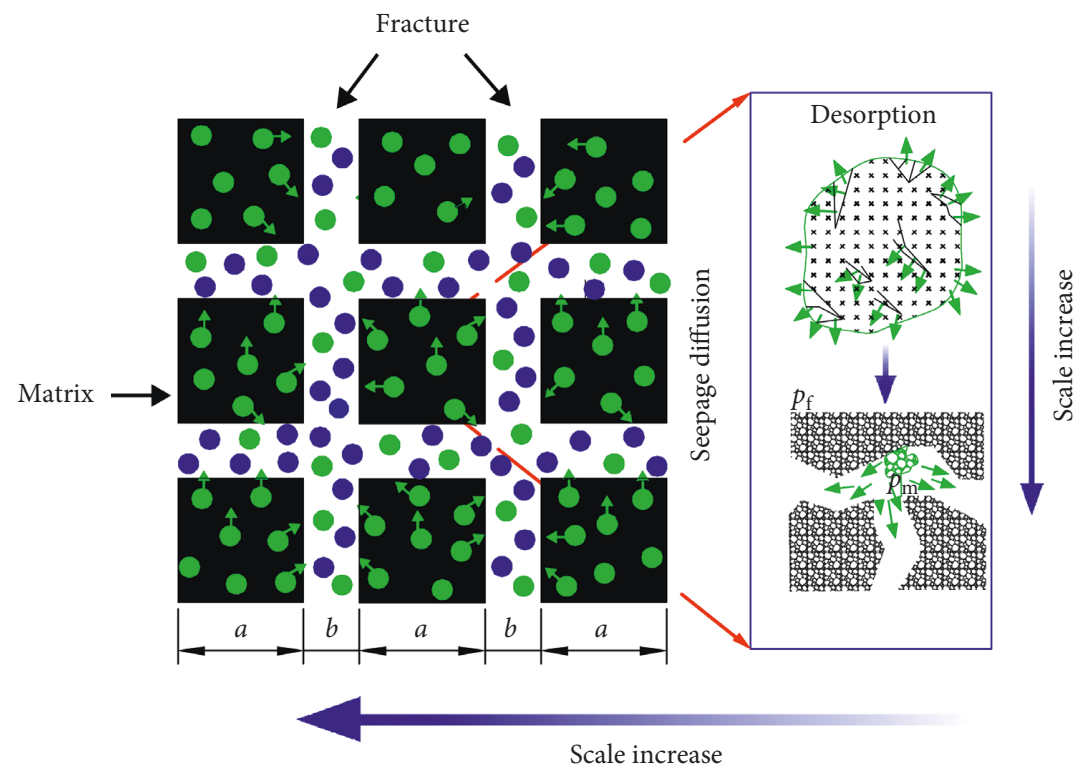

FIgURE 3: Gas migration modes from matrix to fracture.

is saturated by the single-phase gas; (5) gas transportation in the fracture system conforms to Darcy's law, and gas transfer from the matrix system into the fracture system can be divided into seepage and diffusion terms, satisfying Darcy's law and Fick's law, respectively.

\subsection{Governing Equations for a Hydraulic-Mechanical Coupling Model}

2.2.1. Gas Seepage-Diffusion in the Coal Matrix. Gas extraction reduces the pressure near borehole which will break the gas adsorption equilibrium in the coal matrix. The absorbed gas desorbs into the space of pores and transports to the fractures driven by the pressure and concentration gradients via diffusion and seepage accordingly $[34,35]$. Gas in the coal matrix includes adsorbed and free gases and can be defined as

$$
m_{\mathrm{m}}=\phi_{\mathrm{m}} \rho_{\mathrm{g}}+\left(1-\phi_{\mathrm{m}}-\phi_{\mathrm{f}}\right) V_{\mathrm{sg}} \rho_{\mathrm{s}} \rho_{\mathrm{gs}},
$$

where $\phi_{\mathrm{m}}$ is the porosity of the coal matrix, $\rho_{\mathrm{g}}$ is the gas density $\left(\mathrm{kg} / \mathrm{m}^{3}\right), \phi_{\mathrm{f}}$ is the porosity of the fracture, $V_{\mathrm{sg}}$ is the absorbed gas content $\left(\mathrm{m}^{3} / \mathrm{kg}\right), \rho_{\mathrm{s}}$ is the density of the coal skeleton $\left(\mathrm{kg} / \mathrm{m}^{3}\right)$, and $\rho_{\mathrm{gs}}$ is the gas density under standard conditions $\left(\mathrm{kg} / \mathrm{m}^{3}\right)$.

According to the ideal gas law, gas density can be expressed as

$$
\rho_{\mathrm{g}}=\frac{M_{\mathrm{g}}}{R T} p
$$

where $M_{\mathrm{g}}$ is the gas molar mass $(\mathrm{kg} / \mathrm{mol}), R$ is the gas molar constant $(\mathrm{J} /(\mathrm{mol} \cdot \mathrm{K})), p$ is the gas pressure $(\mathrm{MPa})$, and $T$ is the coal seam temperature $(\mathrm{K})$.

The Langmuir equation is used to define the absorbed gas content:

$$
V_{\mathrm{sg}}=\frac{a p_{\mathrm{m}}}{1+b p_{\mathrm{m}}}
$$

where $a$ is the Langmuir volume constant $\left(\mathrm{m}^{3} / \mathrm{kg}\right), b$ is the Langmuir pressure constant $(1 / \mathrm{MPa})$, and $p_{\mathrm{m}}$ is the gas pressure in the coal matrix $(\mathrm{MPa})$.

By introducing the matrix seepage and diffusion terms, the mass conservation equation in the coal matrix can be defined as

$$
\frac{\partial m_{\mathrm{m}}}{\partial t}+\nabla \cdot\left(-\frac{M_{\mathrm{g}} p_{\mathrm{m}}}{R T} \frac{k_{\mathrm{m}}}{\mu} \nabla p_{\mathrm{m}}\right)=-\frac{M_{\mathrm{g}}}{\tau R T}\left(p_{\mathrm{m}}-p_{\mathrm{f}}\right),
$$

where $t$ is the time $(\mathrm{s}) ; p_{\mathrm{f}}$ is the gas pressure $(\mathrm{MPa}) ; \tau$ is the gas desorption time, which reflects the gas diffusion capacity of the coal matrix; $\mu$ is the dynamic viscosity of gas $(\mathrm{Pa} \cdot \mathrm{s}) ; \nabla$ is the Hamiltonian operator; and $k_{\mathrm{m}}$ is the permeability of the coal matrix $\left(\mathrm{m}^{2}\right)$.

Submitting equations (1)-(3) into equation (4), the governing equation for gas transportation in the coal matrix system is obtained as follows:

$$
\begin{aligned}
& \frac{M_{\mathrm{g}}}{R T} \frac{\partial p_{\mathrm{m}} \phi_{\mathrm{m}}}{\partial t}+\frac{M_{\mathrm{g}} p_{\mathrm{s}}}{R T_{\mathrm{s}}} \frac{\partial}{\partial t}\left[\frac{\left(1-\phi_{\mathrm{m}}-\phi_{\mathrm{f}}\right) a b \rho_{\mathrm{s}}}{1+b p_{\mathrm{m}}}\right] \\
& \quad-\nabla \cdot\left(\frac{M_{\mathrm{g}} p_{\mathrm{m}}}{R T} \frac{k_{\mathrm{m}}}{\mu} \nabla p_{\mathrm{m}}\right)=-\frac{M_{\mathrm{g}}}{\tau R T}\left(p_{\mathrm{m}}-p_{\mathrm{f}}\right) .
\end{aligned}
$$

2.2.2. Gas Seepage in Fractures. The coal matrix system provides the mass source for the fracture system. According to the mass conservation, the governing equation of gas seepage in the fracture system considering the Klinkenberg effect can be defined as

$$
\begin{aligned}
& \frac{M_{\mathrm{g}}}{R T}\left(\frac{\partial \phi_{\mathrm{f}}}{\partial t} p_{\mathrm{f}}+\frac{\partial p_{\mathrm{f}}}{\partial t} \phi_{\mathrm{f}}\right)+\nabla \cdot\left(-\frac{M_{\mathrm{g}} p_{\mathrm{f}}}{R T} \frac{k_{\mathrm{f}}}{\mu}\left(1+\frac{m}{p_{\mathrm{f}}}\right) \nabla p_{\mathrm{f}}\right) \\
& \quad=\left(1-\phi_{\mathrm{f}}\right) \frac{M_{\mathrm{g}}}{\tau R T}\left(p_{\mathrm{m}}-p_{\mathrm{f}}\right),
\end{aligned}
$$


where $\rho_{\mathrm{fg}}$ is the gas density in fracture $\left(\mathrm{kg} / \mathrm{m}^{3}\right), k_{\mathrm{f}}$ is the permeability of fracture $\left(\mathrm{m}^{2}\right)$, and $m$ is the Klinkenberg factor $(\mathrm{MPa})$.

2.2.3. Coal Deformation. Coal mass is regarded as the inhomogeneous elastic medium with dual porosity (matrix pores and fractures) and dual permeability (matrix and fracture permeability). Coal deformation reflects the combined actions of gas pressure in the matrix pore and fracture, gas sorption induced stress, and overburden stress, which can be expressed as [36]

$\varepsilon_{i j}=\frac{1}{2 G} \sigma_{i j}-\left(\frac{1}{6 G}-\frac{1}{9 K}\right) \sigma_{k k} \delta_{i j}+\frac{1}{3 K}\left(\alpha_{\mathrm{m}} p_{\mathrm{m}}+\alpha_{\mathrm{f}} p_{\mathrm{f}}\right) \delta_{i j}+\frac{1}{3} \varepsilon_{\mathrm{s}} \delta_{i j}$,

where $\delta_{i j}$ is the Kronecker factor, $\varepsilon_{\mathrm{s}}$ is the sorption induced strain, $G$ is the shear modulus (GPa), $K=E /(1-2 v)$ is the bulk modulus of coal mass (Pa), $E$ is the elastic modulus (GPa), $v$ is the Poisson ratio, $\alpha_{\mathrm{m}}=1-K /\left(a_{n} K_{n}\right)$ and $\alpha_{\mathrm{f}}=1-K / K_{\mathrm{s}}$ are the Biot coefficients for matrix and fracture, $K_{\mathrm{s}}$ is the bulk modulus of the coal skeleton (MPa), $a_{n}$ is the width of the coal matrix (m), and $k_{n}$ is the stiffness of the fracture $(\mathrm{Pa})$.

The Langmuir-type equation is applied to express the relation between sorption-induced strain and gas pressure [37]:

$$
\varepsilon_{\mathrm{s}}=\frac{\varepsilon_{1} p_{\mathrm{m}}}{p_{\mathrm{m}}+p_{\mathrm{l}}}
$$

where $\varepsilon_{1}$ is the maximum sorption-induced strain and $p_{1}$ is the gas pressure corresponding to $50 \%$ of the maximum sorption-induced strain.

The strain-displacement relation of coal is satisfied with the Cauchy formula:

$$
\varepsilon_{i j}=\frac{1}{2}\left(u_{i, j}+u_{j, i}\right)
$$

where $u_{i}$ is the displacement at $i$ direction (m) with $i=x, y, z$.

According to the elastic mechanics, the stress equilibrium relation can be expressed as

$$
\sigma_{i j, j}+F_{i}=0
$$

where $F_{i}$ is the body force of coal.

Submitting equations (8)-(10) into equation (10), we can derive the governing equation of coal deformation:

$$
G u_{i, j j}+\frac{G}{1-2 v} u_{j, j i}-\alpha_{\mathrm{m}} P_{\mathrm{m}, i}-\alpha_{\mathrm{f}} P_{\mathrm{f}, i}-K \varepsilon_{\mathrm{s}, i}+F_{i}=0 .
$$

2.2.4. Coupling Terms. Porosity and permeability play a significant role in the coupling relationship between coal deformation and gas transportation in the coal seam [23, 33]. According to [12], the porosity of the matrix can be defined as

$$
\phi_{\mathrm{m}}=\frac{1}{(1+S)}\left[\phi_{\mathrm{m} 0}\left(1+S_{0}\right)+\alpha_{\mathrm{m}}\left(S-S_{0}\right)\right] \text {, }
$$

where $S=\varepsilon_{\mathrm{v}}+p_{\mathrm{m}} / K_{\mathrm{s}}-\alpha_{T} T-\varepsilon_{\mathrm{s}}, S_{0}=\varepsilon_{\mathrm{v} 0}+p_{\mathrm{m} 0} / K_{\mathrm{s}}-\alpha_{T} T_{0}-\varepsilon_{\mathrm{s} 0}$, $\varepsilon_{\mathrm{v}}$ is the volume strain of coal; and the subscript " 0 " represents the initial value of the corresponding variable.
The cubic law is adopted to establish the relation between matrix porosity and matrix permeability:

$$
\frac{k_{\mathrm{m}}}{k_{\mathrm{m} 0}}=\left\{\frac{1}{(1+S)}\left[\left(1+S_{0}\right)+\frac{\alpha_{\mathrm{m}}\left(S-S_{0}\right)}{\phi_{\mathrm{m} 0}}\right]\right\}^{3} .
$$

Based on the definition of fracture porosity, the change in fracture porosity can be derived as [38-40]

$$
\frac{\phi_{\mathrm{f}}}{\phi_{\mathrm{f} 0}}=1+\frac{\Delta b}{b_{0}} \text {. }
$$

Due to a change in effective stress, the corresponding fracture dilation can be obtained as follows [41, 42]

$$
\Delta b=-\frac{b_{0}}{K_{\mathrm{f}}} \Delta \sigma_{e}=-\frac{3 b_{0}}{\phi_{\mathrm{f} 0}+\left(3 K_{\mathrm{f}} / K\right)}\left(\frac{\varepsilon_{\mathrm{l}} \Delta p_{\mathrm{m}}}{p_{\mathrm{l}}+\Delta p_{\mathrm{m}}}-\varepsilon_{\mathrm{v}}\right) .
$$

Experiments show that the permeability of the coal fracture varies with porosity and is denoted as follows [43]:

$$
\frac{k_{\mathrm{f}}}{k_{\mathrm{f} 0}}=\left(\frac{\phi_{\mathrm{f}}}{\phi_{\mathrm{f} 0}}\right)^{3}=\left[1-\frac{3}{\phi_{\mathrm{f} 0}+\left(3 K_{\mathrm{f}} / K\right)}\left(\frac{\varepsilon_{\mathrm{l}} \Delta p_{\mathrm{m}}}{p_{\mathrm{l}}+\Delta p_{\mathrm{m}}}-\varepsilon_{\mathrm{v}}\right)\right]^{3} \text {. }
$$

The existence of fractures in coal seams often makes the coal permeability show anisotropic characteristics. Considering the anisotropy characteristics, the fracture permeability in the coal seam can be recovered as $[18,38]$

$$
\left\{\begin{aligned}
\frac{k_{\mathrm{f} x}}{k_{\mathrm{f} 0}}= & \frac{1}{2}\left[1-\frac{3}{\phi_{\mathrm{f} 0}+\left(3 K_{\mathrm{f}} / K\right)}\left(\frac{\varepsilon_{\mathrm{l}} \Delta p_{\mathrm{m}}}{p_{\mathrm{l}}+\Delta p_{\mathrm{m}}}-\varepsilon_{y}\right)\right]^{3} \\
& +\frac{1}{2}\left[1-\frac{3}{\phi_{\mathrm{f} 0}+\left(3 K_{\mathrm{f}} / K\right)}\left(\frac{\varepsilon_{\mathrm{l}} \Delta p_{\mathrm{m}}}{p_{\mathrm{l}}+\Delta p_{\mathrm{m}}}-\varepsilon_{z}\right)\right]^{3}, \\
\frac{k_{\mathrm{f} y}}{k_{\mathrm{f} 0}}= & \frac{1}{2}\left[1-\frac{3}{\phi_{\mathrm{f} 0}+\left(3 K_{\mathrm{f}} / K\right)}\left(\frac{\varepsilon_{1} \Delta p_{\mathrm{m}}}{p_{1}+\Delta p_{\mathrm{m}}}-\varepsilon_{x}\right)\right]^{3} \\
& +\frac{1}{2}\left[1-\frac{3}{\phi_{\mathrm{f} 0}+\left(3 K_{\mathrm{f}} / K\right)}\left(\frac{\varepsilon_{\mathrm{l}} \Delta p_{\mathrm{m}}}{p_{\mathrm{l}}+\Delta p_{\mathrm{m}}}-\varepsilon_{z}\right)\right]^{3}, \\
\frac{k_{\mathrm{f} z}=}{k_{\mathrm{f} 0}} & \frac{1}{2}\left[1-\frac{3}{\phi_{\mathrm{f} 0}+\left(3 K_{\mathrm{f}} / K\right)}\left(\frac{\varepsilon_{\mathrm{l}} \Delta p_{\mathrm{m}}}{p_{1}+\Delta p_{e}}-\varepsilon_{x}\right)\right]^{3} \\
& +\frac{1}{2}\left[1-\frac{3}{\phi_{\mathrm{f} 0}+\left(3 K_{\mathrm{f}} / K\right)}\left(\frac{\varepsilon_{\mathrm{l}} \Delta p_{\mathrm{m}}}{p_{\mathrm{l}}+\Delta p_{\mathrm{m}}}-\varepsilon_{y}\right)\right]^{3},
\end{aligned}\right.
$$

where $k_{\mathrm{f} x}, k_{\mathrm{f} y}$, and $k_{\mathrm{f} z}$ are the fracture permeabilities at $x, y$, and $z$ directions, respectively $\left(\mathrm{m}^{2}\right)$.

We assemble equations (5), (6), (11)-(13), and (17) to establish the hydraulic-mechanical coupling model with dualporosity dual-permeability in the coal seam. The bidirectional interactions include the coal deformation (M), gas sorption, and transport $(\mathrm{H})$ fields. The equations are complex nonlinear second-order partial differential equations (PDEs) that are 
difficult to solve analytically [27]. We utilize the finite element (FE) method to obtain a numerical solution.

\section{Modelling of Underground Gas Extraction Based on the Proposed Model}

3.1. Parameter Setting and Geometry Model. Changping Coal Mine is located in Jincheng, Shanxi Province, China. No. 3 coal seam in the Shanxi formation with a uniform thickness $(5 \sim 6 \mathrm{~m})$ is rich in mine gas $\left(8.27 \sim 21.54 \mathrm{~m}^{3} / t\right)$, which may cause a gas dynamic disaster such as gas outburst or gas explosion. We utilize the proposed mathematical model to simulate the characteristics of gas migration in the coal seam during underground gas extraction using COMSOL Multiphysics. The block with $20 \mathrm{~m}$ (length) $\times 15 \mathrm{~m}$ (width) $\times 5 \mathrm{~m}$ (height) is used as the geometry model. The geometry model and boundary conditions are shown in Figure 4.

For coal deformation, the basal boundary is zero deformation with slip conditions applied to the surrounding boundaries and overburden loading on the upper boundary $[25,26]$. For the gas flow, a constant pressure of $75 \mathrm{kPa}$ (suction pressure of $26 \mathrm{kPa}$ ) is applied to the extraction well. Other boundaries are insulated for gas flow. The initial gas pressure, matrix permeability, and fracture permeability are set as $1.52 \mathrm{MPa}, 5.14 \times 10^{-19} \mathrm{~m}^{2}$, and $2.18 \times 10^{-17} \mathrm{~m}^{2}$, respectively. At the beginning, the coal seam is in free stress state. The related parameters used in gas extraction simulation are shown in Table 1.

The anisotropy coefficient of fracture permeability in the coal seam is defined as follows:

$$
\lambda=1-\frac{k_{\mathrm{f} z}}{k_{\mathrm{f} x}},
$$

where the anisotropy coefficient ranges from 0 to 1 . The larger the value, the more obvious the anisotropy will be.

In order to analyze the relationship between permeability anisotropy and gas production, permeability evolution, matrix seepage term, and gas pressure, three scenarios of different permeability anisotropy coefficients were simulated, namely, $\lambda=0,0.3$, and 0.6 , respectively.

\subsection{Modelling Results of Underground Gas Extraction}

3.2.1. Relationship between Anisotropy and Gas Pressure. The evolution of parameters, such as gas pressure and permeability, on reference point $\mathrm{A}$ is quantitatively analyzed to illustrate the impact of permeability anisotropy on gas extraction. Figure 5 shows the location of reference points A $(11.5,0,2), \mathrm{B}(10,0,4)$, and C $(18,0,4)$.

Different anisotropy coefficients of permeability correspond to different gas pressure distribution characteristics. To compare the results of gas extraction of different anisotropic permeabilities, the anisotropic coefficient of 0 is selected as the standard scenario. In Figure 6, with the increase of extraction time, the gas pressure at reference point A decreases gradually and the decreasing speed decreases slowly. Greater anisotropy coefficient of fracture permeability leads to lower gas extraction

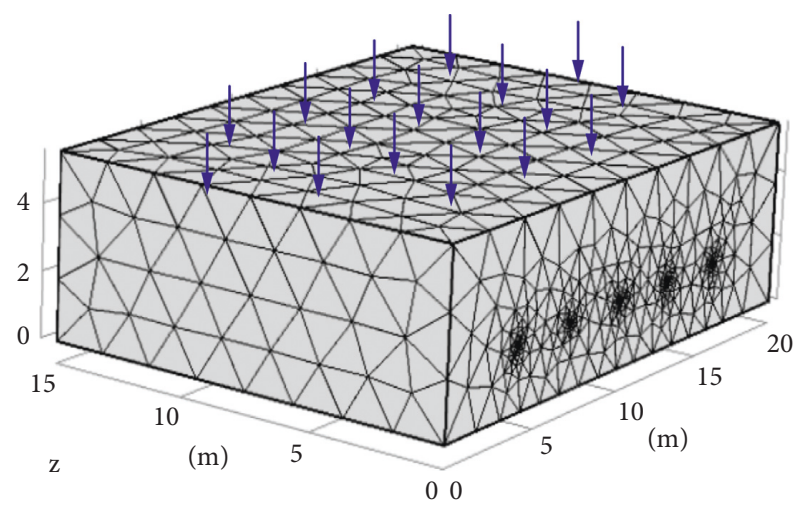

FIgURE 4: Geometry model and boundary conditions.

efficiency. This phenomenon becomes more obvious when the extraction time continuously increases. Taking the anisotropic coefficient of 0.3 and 0.6 as an example, the extraction efficiencies are 0.83 and 0.64 after 90 days' gas extraction, respectively.

Figure 7 shows variation in the pressure gradient of the matrix-fracture system with extraction time. The pressure gradient increases first and then decreases. At the initial stage of gas extraction, the pressure gradient of the matrixfracture system is more significant than that in the late stage because of the anisotropy of fracture permeability. Due to the larger gas pressure gradient at the beginning, the rate of gas desorption from the coal matrix is faster. However, at the later stage of extraction, the pressure gradient within the matrix-fracture system of greater anisotropic coefficient is larger than that of smaller anisotropic coefficient.

3.2.2. Evolution of Permeability. Figures $8-10$ present the matrix and fracture permeability evolution under different anisotropic coefficients. With the increase in gas extraction time, both matrix permeability and fracture permeability increase in a polynomial form. The increase rate of matrix permeability is greater than that of fracture permeability. The fracture permeability in $x$ and $y$ directions have the same increasing trend, indicating that the influence of gas extraction on them is basically similar. The magnitudes of the matrix permeability ratio are $1.65,1.6$, and 1.57 corresponding to anisotropy coefficients $0,0.3$, and 0.6 , respectively.

3.2.3. Influence of the Matrix Seepage Term on Gas Pressure. The adsorbed gas desorbs to form free gas in the matrix system and then transports to the fracture system by means of diffusion and seepage. The proportion of matrix seepage flux to total flux from the matrix to fracture system is analyzed, as well as the influence of matrix seepage on gas extraction.

Figure 11 shows that the seepage proportion increases rapidly at first and then decreases and eventually tends to be flat. The more obvious the permeability anisotropy is, the smaller the exchange capacity between matrix system and fracture system is. When the anisotropy coefficient is 0 , the seepage flow at point $\mathrm{A}$ decreases with the increase of gas 
TABLE 1: Related parameters for simulation of underground gas extraction.

\begin{tabular}{lcc}
\hline Parameter & Value & Source \\
\hline Initial porosity of the matrix, $\phi_{\mathrm{m} 0}$ & 0.045 & Experiments \\
Gas dynamic viscosity, $\mu(\mathrm{Pa} \cdot \mathrm{s})$ & $1.84 \times 10^{-5}$ & [38] \\
Langmuir volume constants, $a\left(\mathrm{~m}^{3} \cdot \mathrm{kg}^{-1}\right)$ & 2.45 & Experiments \\
Langmuir pressure constants, $b\left(\mathrm{MPa}^{-1}\right)$ & 0.018 & Experiments \\
Width of the coal matrix, $a_{n}(\mathrm{~m})$ & $2 \times 10^{-2}$ & Estimation \\
Initial fracture porosity, $\phi_{\mathrm{f} 0}$ & 0.015 & Experiments \\
Desorption time, $\tau($ days $)$ & 9.2 & {$[12]$} \\
Poisson's ratio, $v$ & 0.28 & Experiments \\
Klinkenberg factor, $\mathrm{m}(\mathrm{MPa})$ & 0.76 & [26] \\
Coal seam temperature, $T(\mathrm{~K})$ & 305.15 & Field test \\
Strain constant, $\varepsilon_{1}$ & 0.0083 & {$[27]$} \\
Coal skeleton density, $\rho_{\mathrm{s}}\left(\mathrm{kg} \cdot \mathrm{m}^{-3}\right)$ & 1420 & Experiments \\
Pressure constant, $p_{1}(\mathrm{MPa})$ & 3.55 & {$[27]$} \\
Initial gas pressure, $p(\mathrm{MPa})$ & 1.52 & Field test \\
\hline
\end{tabular}

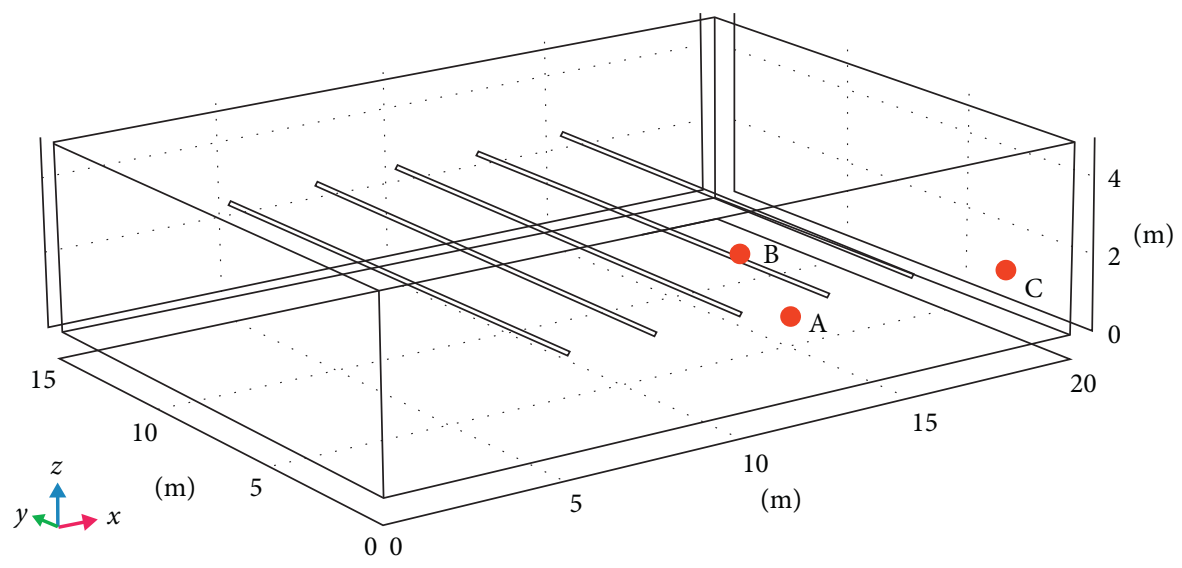

FIGURE 5: Location of reference points.

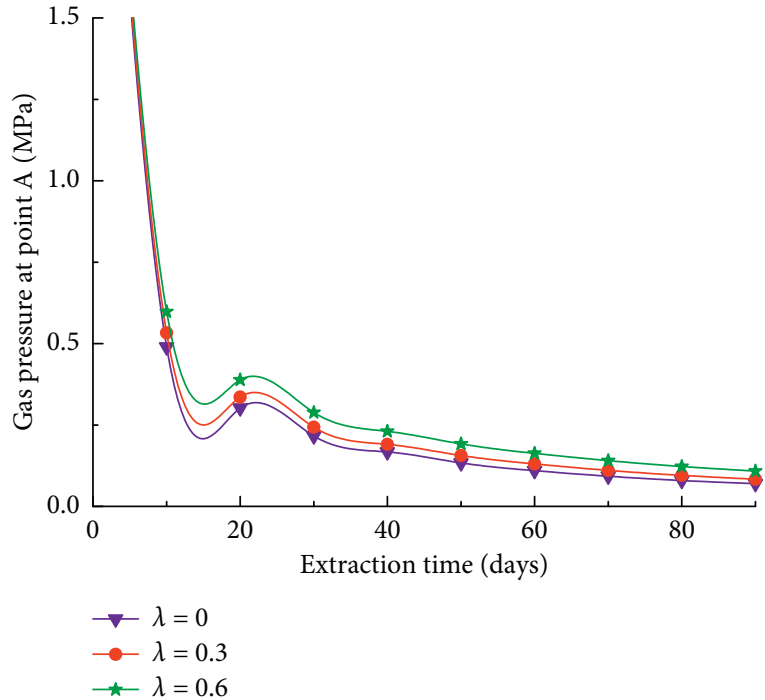

(a)

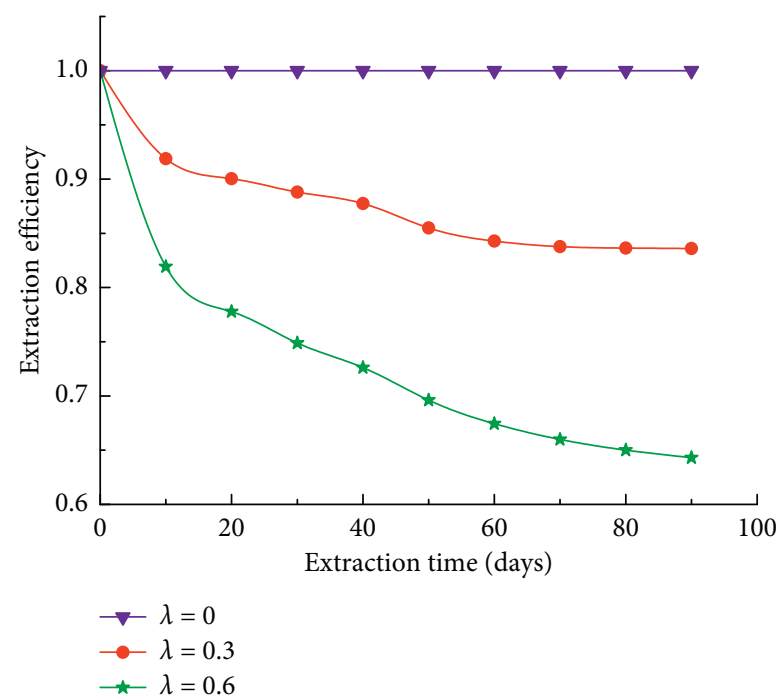

(b)

FIGURE 6: (a) Gas pressure and (b) extraction efficiency at point A for different anisotropic coefficients.

extraction, and the seepage proportion approaches $0.1 \%$ from $0.35 \%$ at the initial stage. When $\lambda=0.3$, the seepage flow at point A shows a uniform upward trend and finally a stable trend, and the seepage flow proportion is ultimately $0.09 \%$. When $\lambda=0.6$, the seepage proportion of point $\mathrm{A}$ is up to $1.3 \%$, and the seepage flow proportion is finally $1 \%$. 


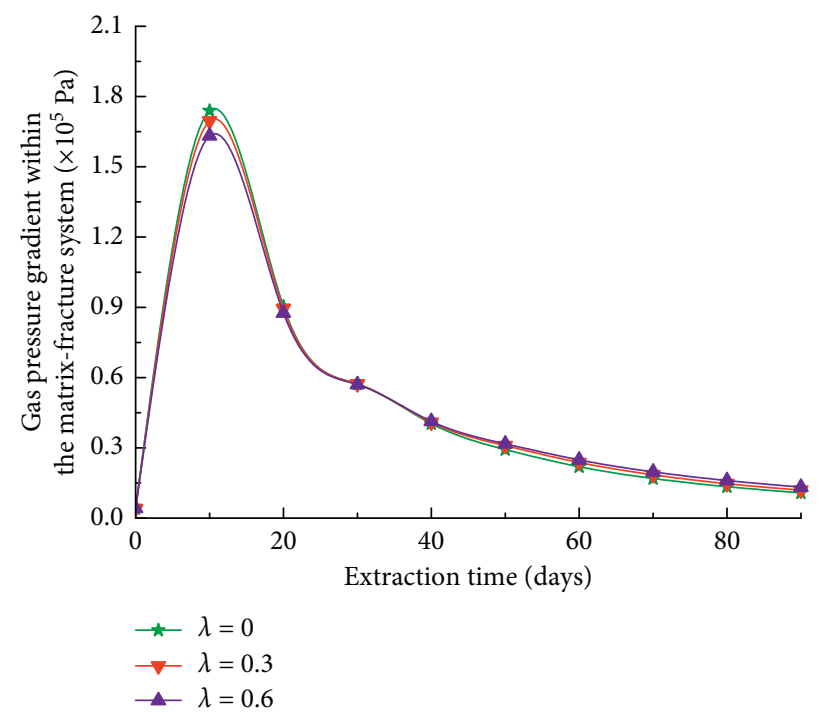

FIGURE 7: Gas pressure gradient within the matrix-fracture system.

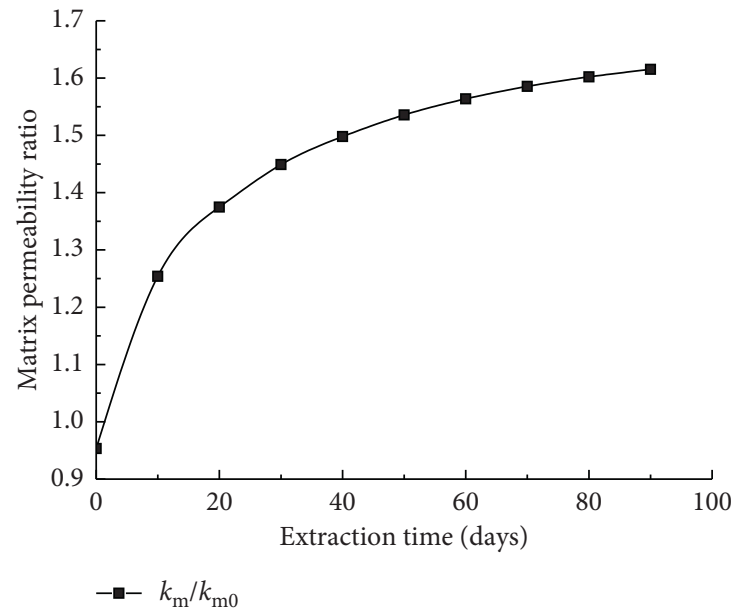

(a)

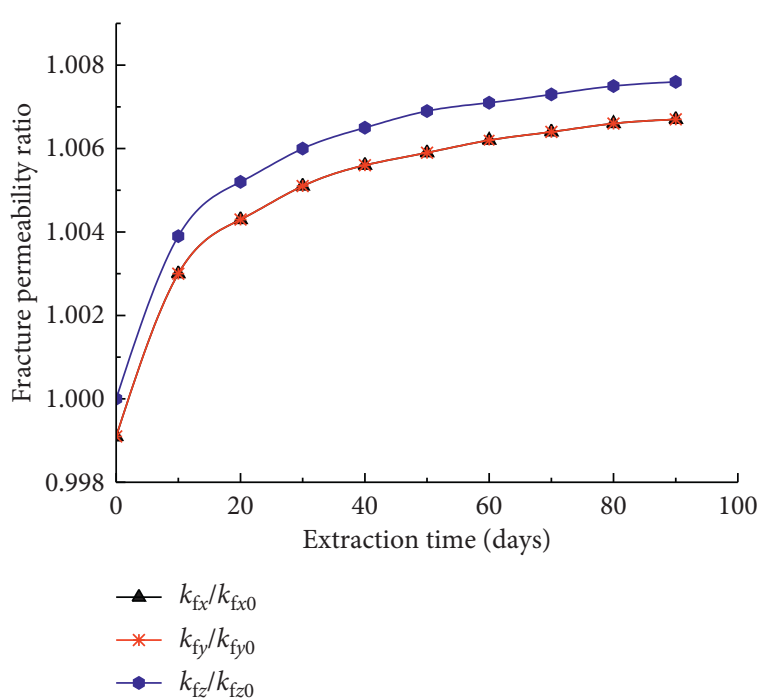

(b)

FIgURE 8: Permeability evolution with the anisotropic coefficient $\lambda=0$. (a) Matrix permeability. (b) Fracture permeability.

It can be inferred that the maximum proportion of seepage flux increases with the increase of anisotropic coefficient initially. However, in the middle and late stage of extraction, the proportion of seepage flux to total flux will not change.

To clarify the influence of the matrix seepage term on gas extraction, the matrix seepage term was removed from the proposed governing equation of gas migration in the coal matrix:

$$
\begin{aligned}
& \frac{M_{\mathrm{g}}}{R T} \frac{\partial p_{\mathrm{m}} \phi_{\mathrm{m}}}{\partial t}+\frac{M_{\mathrm{g}}}{R T_{\mathrm{s}}} \frac{\partial}{\partial t}\left[\left(1-\phi_{\mathrm{m}}-\phi_{\mathrm{f}}\right) \frac{a b \rho_{\mathrm{s}} p_{\mathrm{s}}}{1+b p_{\mathrm{m}}}\right] \\
& \quad=-\frac{M_{\mathrm{g}}}{\tau R T}\left(p_{\mathrm{m}}-p_{\mathrm{f}}\right) .
\end{aligned}
$$

Equations (6), (11)-(13), (17), and (19) were assembled to establish the hydraulic-mechanical coupling model with single permeability. This single-permeability model was compared with the dual-permeability model by simulation of underground gas extraction.

It is generally believed that the amount of gas migration from the matrix system to the fracture system is constant. The permeability of the matrix seepage term is too small that the gas is difficult to seepage into the fracture system. Therefore, the calculation result of the dual-porosity and single-permeability model is larger generally. In this paper, gas pressure under the same anisotropic coefficient $(\lambda=0.6)$ is used to characterize the difference between the dualporosity dual-permeability model and dual-porosity singlepermeability model, as well as the influence of the seepage 


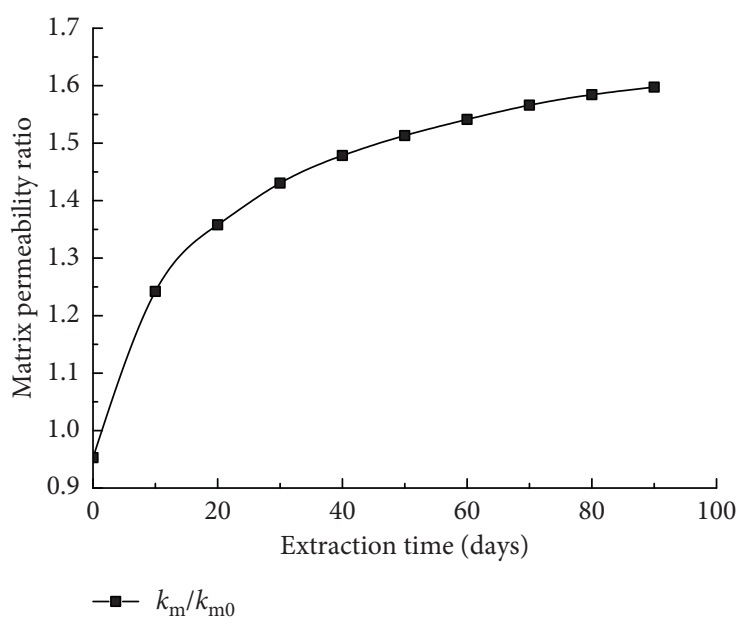

(a)

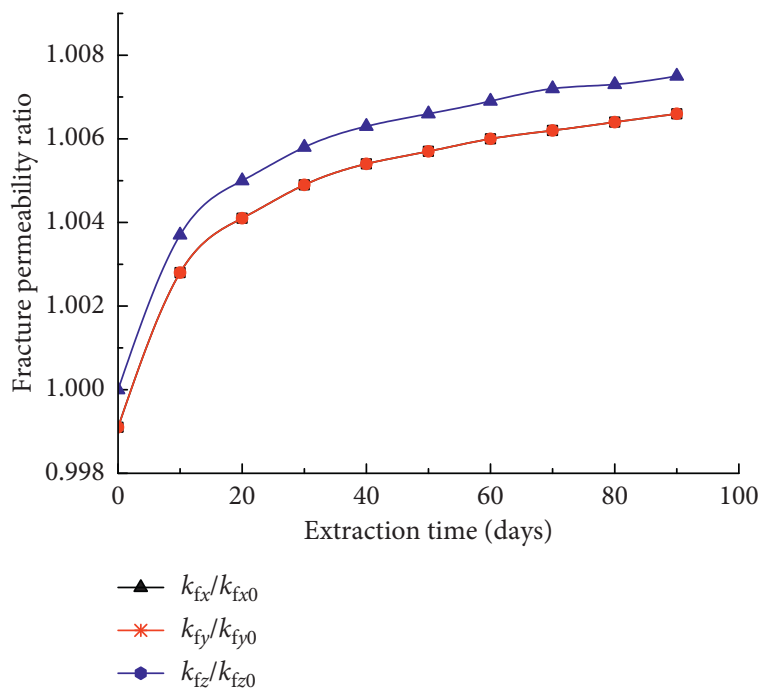

(b)

Figure 9: Permeability evolution with the anisotropic coefficient $\lambda=0.3$. (a) Matrix permeability. (b) Fracture permeability.

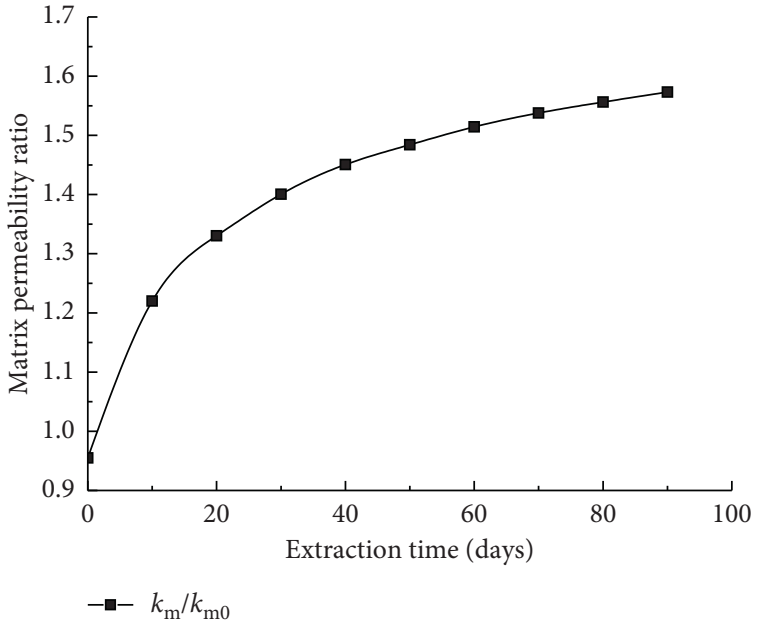

(a)

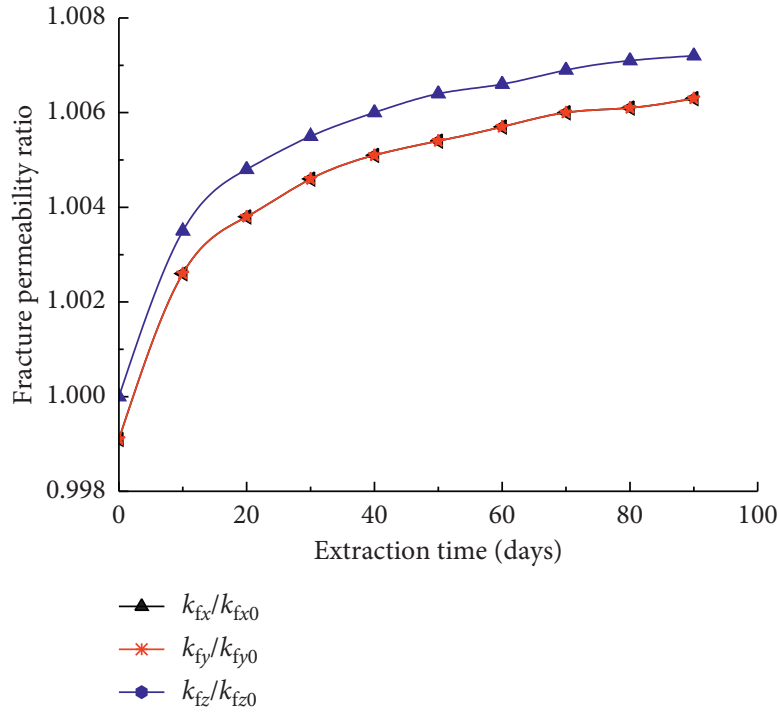

(b)

FIGURE 10: Permeability evolution with the anisotropic coefficient $\lambda=0.6$. (a) Matrix permeability. (b) Fracture permeability.

term on gas extraction. Parameters on three points are discussed, with locations $\mathrm{A}(11.5,0,2), \mathrm{B}(10,0,4)$, and $\mathrm{C}(18$, $0,4)$. To accurately analyze the seepage term, the duration is $360 \mathrm{~h}$ with an interval of $10 \mathrm{~h}$.

Figure 12 presents the gas pressure distribution and pressure ratio of dual-permeability to single-permeability model. A cosine fluctuation shape is found on the curves in the early stage of gas extraction. The pressure ratio decreases first and then increases followed by a decrease. With the continuous gas extraction, the gas pressure ratio of dualpermeability model decreases obviously, while the pressure gradient within the dual-permeability and single-permeability models at the same position increases. The lowest gas pressure ratios of dual-permeability model to single-permeability model at Points A, B, and C are 0.98739, 0.98966, and 0.99369 , respectively. Hence, the seepage term in the coal matrix can enhance the efficiency of gas extraction and plays a promoting role in the specific stage of gas extraction.

\section{Conclusions}

(1) The NMR curve of pore diameter distribution in the coal sample has an independent bimodal shape with large difference between the two peaks. The micropore is the main pore structure, accounting for $91 \% \sim 96 \%$ of all pores. The micropores and medium 


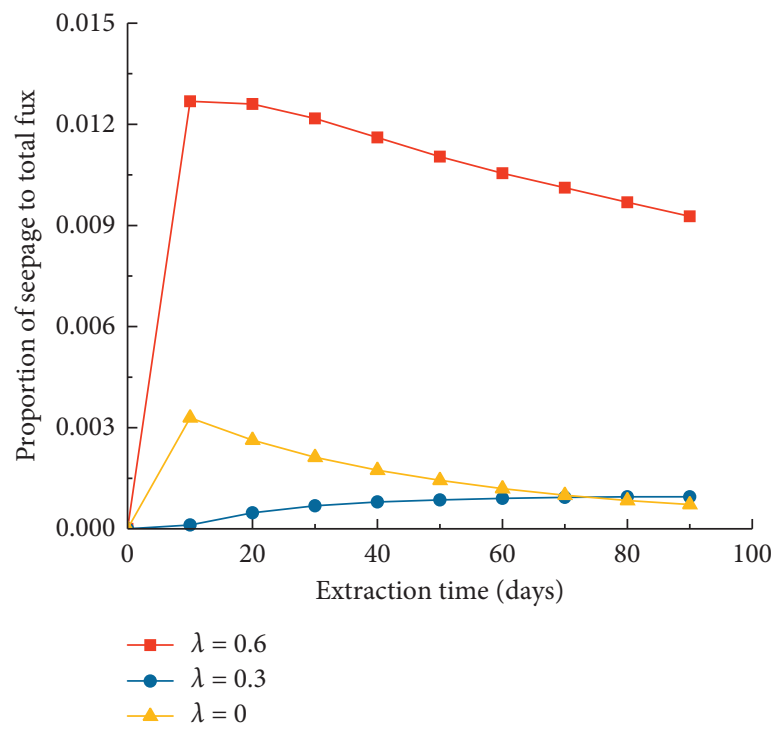

Figure 11: Evolution of proportion of matrix seepage flux to total flux from the matrix to fracture.

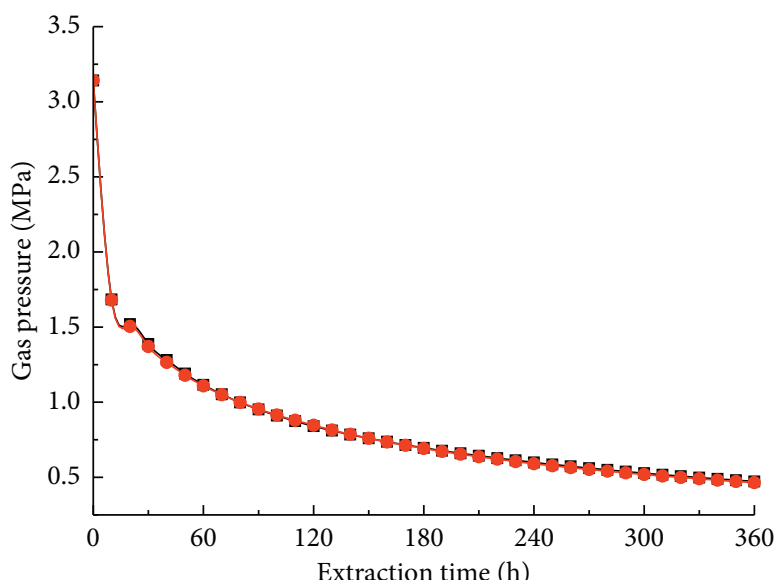

Extraction time (h)

$\rightarrow$ Single permeability

$\rightarrow$ Dual permeability

(a)

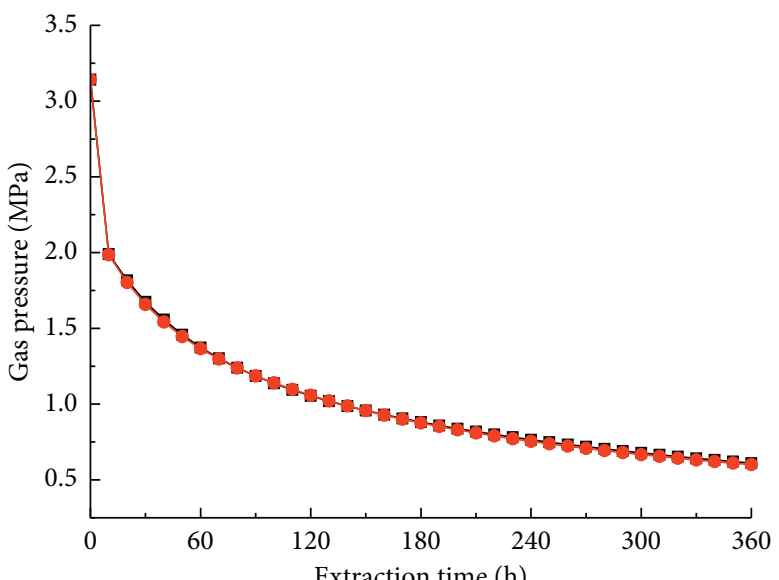

- Single permeability

- - Dual permeability

(c)

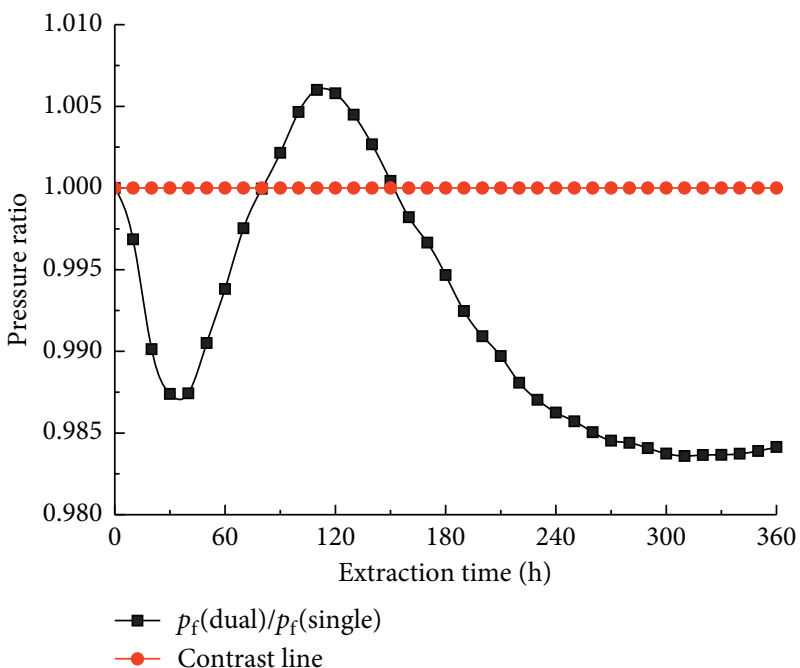

(b)

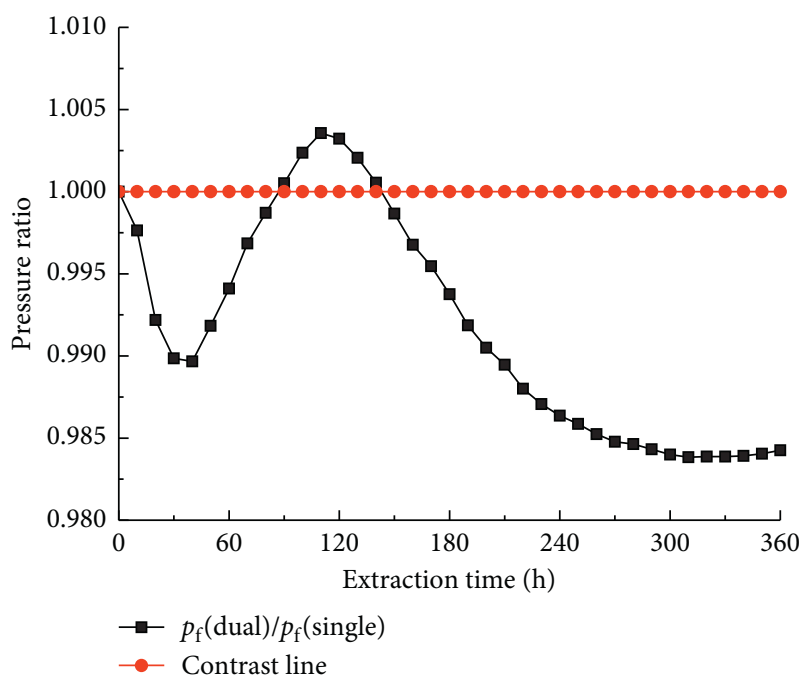

(d)

Figure 12: Continued. 


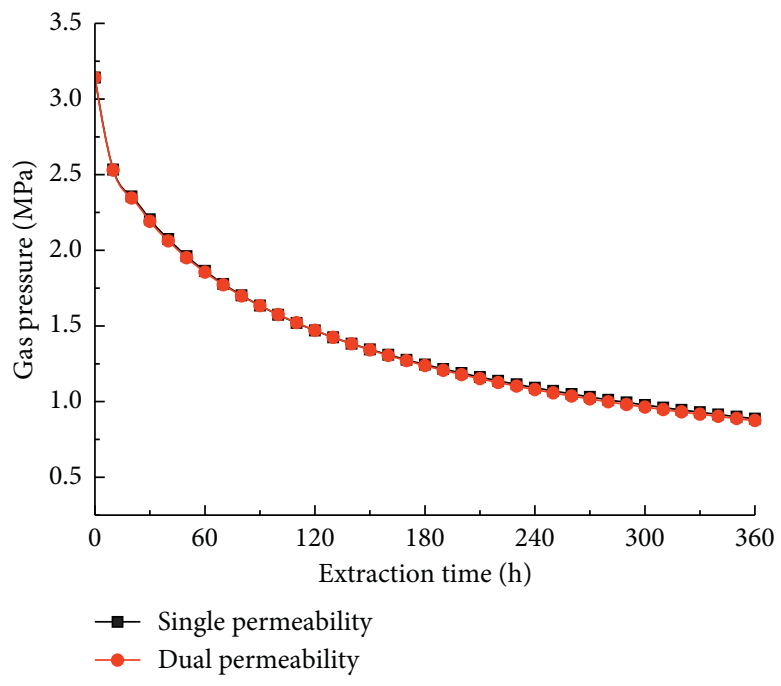

(e)

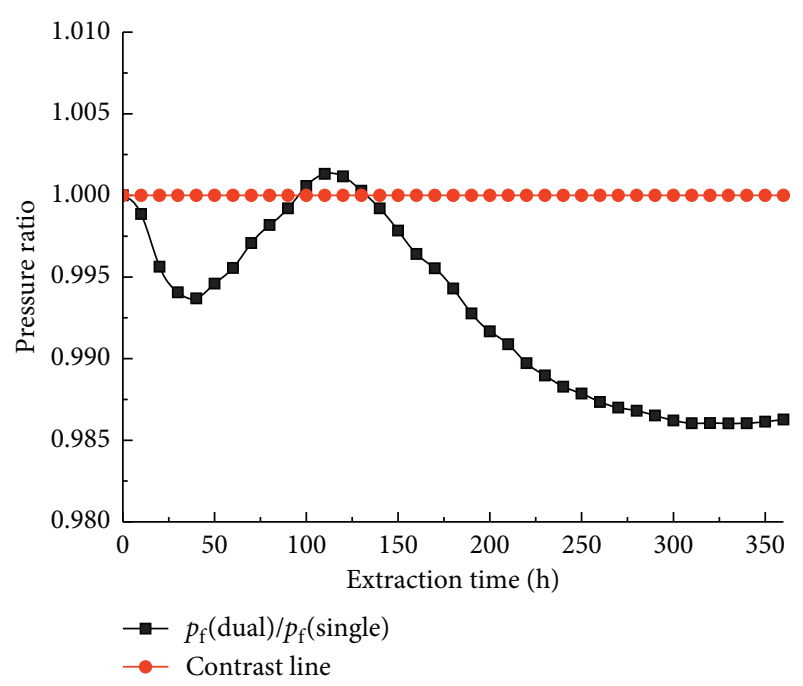

(f)

Figure 12: Comparative analysis of gas pressure in two models at different locations. Gas pressure and pressure ratio at (a) Point A, (b) Point $\mathrm{B}$, and (c) Point C.

pores are not connected. The pore throat aperture which is smaller than $0.1 \mu \mathrm{m}$ accounts for more than $90 \%$ of all pore throats. This evidences the assumption of the dual-porosity dual-permeability model.

(2) Considering the mass exchange within the pore and fracture, the matrix permeability is proposed. The hydraulic-mechanical coupling model considering the fracture anisotropy and matrix seepage term is constructed for underground gas extraction simulation.

(3) The proposed hydraulic-mechanical coupling model with dual-porosity dual-permeability is applied to simulate gas extraction of No. 3 coal seam in Changping Coal Mine. The pressure gradient within matrix-fracture increases first and then decreases. The pressure gradient and anisotropic coefficient are proportional with extraction time in the initial stage. The increased rate of matrix permeability is larger than that of fracture permeability. The matrix seepage term will promote gas extraction at a specific stage of gas extraction.

(4) The seepage and diffusion flux are proportional to the anisotropy coefficient. The proportion of matrix seepage flux increases first and then decreases to a certain value. The simulation results of No. 3 coal seam in Changping Coal Mine show that the matrix seepage flux accounts for $0.1 \%$ of the total flux.

The hydraulic-mechanical coupling model with dualporosity and anisotropy characteristics developed in this work not only offers useful framework to investigate important technical challenges associated with underground mine gas extraction but can also be applied to other forms of surface coalbed methane and shale gas development, as well as $\mathrm{CO}_{2}$ geological sequestration in unconventional reservoirs.

\section{Data Availability}

The data used in this article were from the simulation results by Comsol Mutiphysics. The equations were written as Comsol codes independently to conduct these simulations. The data are available from the corresponding author upon request.

\section{Conflicts of Interest}

The authors declared no potential conflicts of interest with respect to the research, authorship, and/or publication of this article.

\section{Acknowledgments}

This work was financially supported by the National Natural Science Foundation of China (Grant no. 51504127) and the Open Projects of Research Center of Coal Resources Safe Mining and Clean Utilization, Liaoning (Grant no. LNTU17KF06).

\section{References}

[1] J. C. Pashin, "Stratigraphy and structure of coalbed methane reservoirs in the United States: an overview," International Journal of Coal Geology, vol. 35, no. 1-4, pp. 209-240, 1998.

[2] I. Palmer, "Coalbed methane completions: a world view," International Journal of Coal Geology, vol. 82, no. 3-4, pp. 184-195, 2010.

[3] Z. Zhou, X. Cai, D. Ma, L. Chen, S. Wang, and L. Tan, "Dynamic tensile properties of sandstone subjected to wetting and drying cycles," Construction and Building Materials, vol. 182, pp. 215-232, 2018.

[4] M. Sayyafzadeh, A. Keshavarz, A. R. M. Alias, K. A. Dong, and M. Manser, "Investigation of varying-composition gas injection for coalbed methane recovery enhancement: a 
simulation-based study," Journal of Natural Gas Science and Engineering, vol. 27, pp. 1205-1212, 2015.

[5] D. Ma, M. Rezania, H.-S. Yu, and H.-B. Bai, "Variations of hydraulic properties of granular sandstones during water inrush: effect of small particle migration," Engineering Geology, vol. 217, pp. 61-70, 2017.

[6] J. Sobczyk, "The influence of sorption processes on gas stresses leading to the coal and gas outburst in the laboratory conditions," Fuel, vol. 90, no. 3, pp. 1018-1023, 2011.

[7] M. B. D. Aguado and C. G. Nicieza, "Control and prevention of gas outbursts in coal mines, Riosa-Olloniego coalfield, Spain," International Journal of Coal Geology, vol. 69, no. 4, pp. 253-266, 2007.

[8] T. Ren, G. Wang, Y. Cheng, and Q. Qi, "Model development and simulation study of the feasibility of enhancing gas drainage efficiency through nitrogen injection," Fuel, vol. 194, pp. 406-422, 2017.

[9] D. Ma, X. Cai, Q. Li, and H. Duan, "In-situ and numerical investigation of groundwater inrush hazard from grouted karst collapse pillar in longwall mining," Water, vol. 10, no. 9, p. 1187, 2018.

[10] Z. Zhou, X. Cai, D. Ma et al., "Water saturation effects on dynamic fracture behavior of sandstone," International Journal of Rock Mechanics and Mining Sciences, vol. 114, pp. 46-61, 2019.

[11] T. Liu, B. Lin, Q. Zou, and C. Zhu, "Microscopic mechanism for enhanced coal bed methane recovery and outburst elimination by hydraulic slotting: a case study in yangliu mine, China," Greenhouse Gases: Science and Technology, vol. 6, no. 5, pp. 597-614, 2016.

[12] S. Li, C. Fan, J. Han, M. Luo, Z. Yang, and H. Bi, "A fully coupled thermal-hydraulic-mechanical model with two-phase flow for coalbed methane extraction," Journal of Natural Gas Science and Engineering, vol. 33, pp. 324-336, 2016.

[13] T. Bi, B. Lin, W. Yang, C. Zhai, and T. Liu, "Coal permeability evolution and gas migration under non-equilibrium state," Transport in Porous Media, vol. 118, no. 3, pp. 393-416, 2017.

[14] T. Liu, B. Lin, and W. Yang, "Impact of matrix-fracture interactions on coal permeability: model development and analysis," Fuel, vol. 207, pp. 522-532, 2017.

[15] G. Yin, B. Deng, M. Li et al., "Impact of injection pressure on $\mathrm{CO}_{2}$-enhanced coalbed methane recovery considering mass transfer between coal fracture and matrix," Fuel, vol. 196, pp. 288-297, 2017.

[16] C. Zhang, J. Liu, Y. Zhao, L. Zhang, and J. Guo, "A fluid-solid coupling method for the simulation of gas transport in porous coal and rock media," Energy Science \& Engineering, 2019.

[17] L. D. Connell, "Coupled flow and geomechanical processes during gas production from coal seams," International Journal of Coal Geology, vol. 79, no. 1-2, pp. 18-28, 2009.

[18] Y. Wu, J. Liu, D. Elsworth, X. Miao, and X. Mao, "Development of anisotropic permeability during coalbed methane production," Journal of Natural Gas Science and Engineering, vol. 2, no. 4, pp. 197-210, 2010.

[19] J. Rutqvist, Y.-S. Wu, C.-F. Tsang, and G. Bodvarsson, "A modeling approach for analysis of coupled multiphase fluid flow, heat transfer, and deformation in fractured porous rock," International Journal of Rock Mechanics and Mining Sciences, vol. 39, no. 4, pp. 429-442, 2002.

[20] W. C. Zhu, J. Liu, J. C. Sheng, and D. Elsworth, “Analysis of coupled gas flow and deformation process with desorption and Klinkenberg effects in coal seams," International Journal of Rock Mechanics and Mining Sciences, vol. 44, no. 7, pp. 971-980, 2007.
[21] G. Hu, H. Wang, X. Fan, Z. Yuan, and S. Hong, "Mathematical model of coalbed gas flow with Klinkenberg effects in multiphysical fields and its analytic solution," Transport in Porous Media, vol. 76, no. 3, pp. 407-420, 2009.

[22] W. C. Zhu, C. H. Wei, J. Liu, H. Y. Qu, and D. Elsworth, “A model of coal-gas interaction under variable temperatures," International Journal of Coal Geology, vol. 86, no. 2-3, pp. 213-221, 2011.

[23] C. Fan, M. Luo, S. Li, H. Zhang, Z. Yang, and Z. Liu, "A thermo-hydro-mechanical-chemical coupling model and its application in acid fracturing enhanced coalbed methane recovery simulation," Energies, vol. 12, no. 4, p. 626, 2019.

[24] T. Teng, Y. Zhao, F. Gao, J. G. Wang, and W. Wang, "A fully coupled thermo-hydro-mechanical model for heat and gas transfer in thermal stimulation enhanced coal seam gas recovery," International Journal of Heat and Mass Transfer, vol. 125, pp. 866-875, 2018.

[25] C. Fan, S. Li, M. Luo, Z. Yang, and T. Lan, "Numerical simulation of hydraulic fracturing in coal seam for enhancing underground gas drainage," Energy Exploration \& Exploitation, vol. 37, no. 1, pp. 166-193, 2019.

[26] C. Fan, D. Elsworth, S. Li et al., "Modelling and optimization of enhanced coalbed methane recovery using $\mathrm{CO}_{2} / \mathrm{N}_{2}$ mixtures," Fuel, vol. 253, pp. 1114-1129, 2019.

[27] C. Fan, D. Elsworth, S. Li, L. Zhou, Z. Yang, and Y. Song, "Thermo-hydro-mechanical-chemical couplings controlling $\mathrm{CH}_{4}$ production and $\mathrm{CO}_{2}$ sequestration in enhanced coalbed methane recovery," Energy, vol. 173, pp. 1054-1077, 2019.

[28] S. G. Cao, Y. B. Liu, Y. Li, L. Q. Zhang, and J. Wang, "Research and development of testing apparatus of coal solid-gas coupled meso-mechanics," Chinese Journal of Rock Mechanics and Engineering, vol. 28, no. 8, pp. 1681-1690, 2009.

[29] Y. Yao, D. Liu, Y. Che, D. Tang, S. Tang, and W. Huang, "Petrophysical characterization of coals by low-field nuclear magnetic resonance (NMR)," Fuel, vol. 89, no. 7, pp. 1371-1380, 2010.

[30] K. P. Zhou, L. J. Li, Y. J. Xu, and Y. M. Zhang, "Measurement of rock pore structure based on NMR technology," Journal of Central South University (Science and Technology), vol. 43, no. 12, pp. 4796-4800, 2012.

[31] H. K. Ren, A. M. Wang, C. F. Li, D. Y. Cao, and Y. C. Wei, "Study on porosity characteristics of low-rank coal reservoirs based on nuclear magnetic resonance technology," Coal Science Technology, vol. 45, no. 4, pp. 143-148, 2017.

[32] K. Fan, Y. Li, D. Elsworth et al., "Three stages of methane adsorption capacity affected by moisture content," Fuel, vol. 231, pp. 352-360, 2018.

[33] G. Wang, K. Wang, S. Wang, D. Elsworth, and Y. Jiang, "An improved permeability evolution model and its application in fractured sorbing media," Journal of Natural Gas Science and Engineering, vol. 56, pp. 222-232, 2018.

[34] S. Durucan, M. Ahsanb, and J.-Q. Shia, "Matrix shrinkage and swelling characteristics of European coals," Energy Procedia, vol. 1, no. 1, pp. 3055-3062, 2009.

[35] K. T. Lim and K. Aziz, "Matrix-fracture transfer shape factors for dual-porosity simulators," Journal of Petroleum Science \& Engineering, vol. 13, no. 3-4, pp. 169-178, 1995.

[36] D. Chen, Z. Pan, J. Liu, and L. D. Connell, "An improved relative permeability model for coal reservoirs," International Journal of Coal Geology, vol. 109-110, pp. 45-57, 2013.

[37] P. Thararoop, Z. T. Karpyn, and T. Ertekin, "Development of a multi-mechanistic, dual-porosity, dual-permeability, numerical flow model for coalbed methane reservoirs," Journal 
of Natural Gas Science and Engineering, vol. 8, pp. 121-131, 2012.

[38] Y. Wu, J. Liu, D. Elsworth, Z. Chen, L. Connell, and Z. Pan, "Dual poroelastic response of a coal seam to $\mathrm{CO}_{2}$ injection," International Journal of Greenhouse Gas Control, vol. 4, no. 4, pp. 668-678, 2010.

[39] R. Shi, J. Liu, M. Wei, D. Elsworth, and X. Wang, "Mechanistic analysis of coal permeability evolution data under stresscontrolled conditions," International Journal of Rock Mechanics and Mining Sciences, vol. 110, pp. 36-47, 2018.

[40] G. Wang, C. Jiang, J. Shen, D. Han, and X. Qin, "Deformation and water transport behaviors study of heterogenous coal using CT-based 3D simulation," International Journal of Coal Geology, vol. 211, article 103204, 2019.

[41] H. Li, S. Shi, J. Lu, Q. Ye, Y. Lu, and X. Zhu, "Pore structure and multifractal analysis of coal subjected to microwave heating," Powder Technology, vol. 346, pp. 97-108, 2019.

[42] H. Li, S. Shi, B. Lin et al., "A fully coupled electromagnetic, heat transfer and multiphase porous media model for microwave heating of coal," Fuel Processing Technology, vol. 189, pp. 49-61, 2019.

[43] Z. Pan and L. D. Connell, "Modelling permeability for coal reservoirs: a review of analytical models and testing data," International Journal of Coal Geology, vol. 92, pp. 1-44, 2012. 


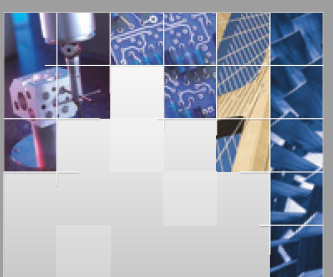

\section{Enfincering}
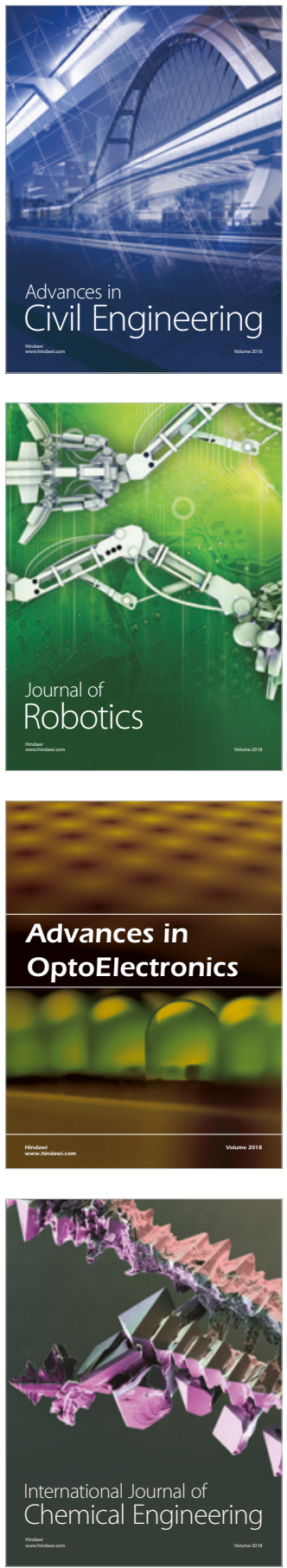

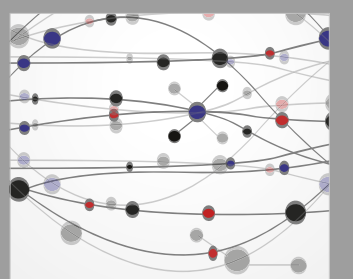

\section{Rotating \\ Machinery}

The Scientific World Journal

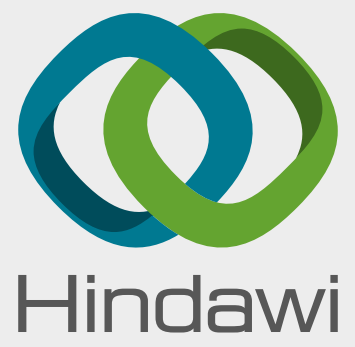

Submit your manuscripts at

www.hindawi.com
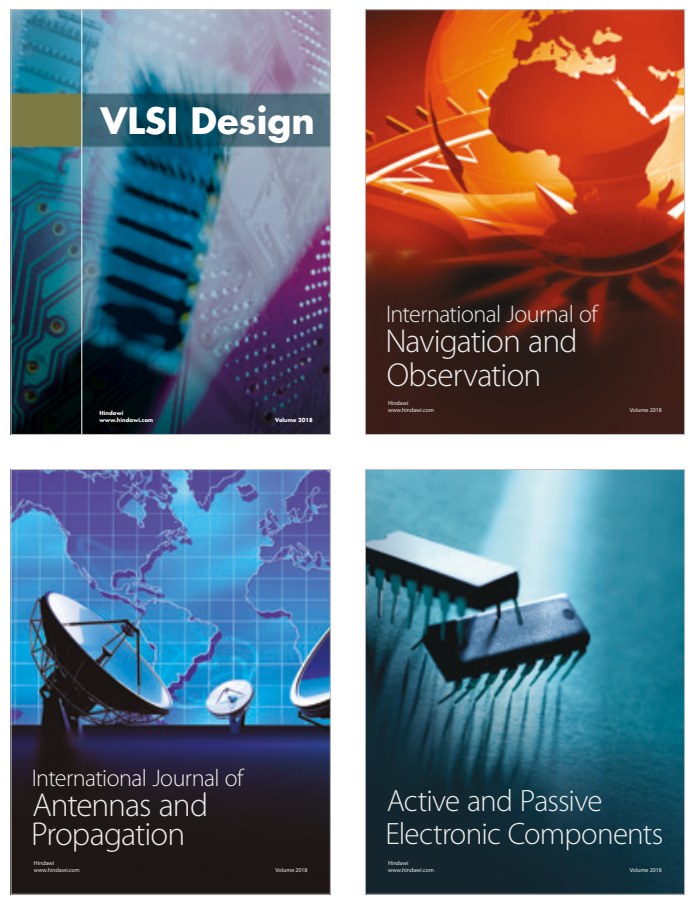
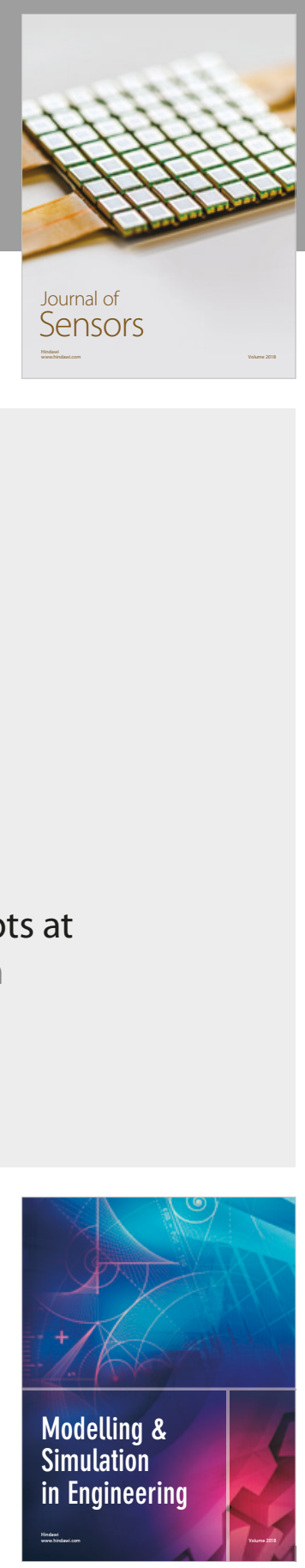

\section{Advances \\ Multimedia}
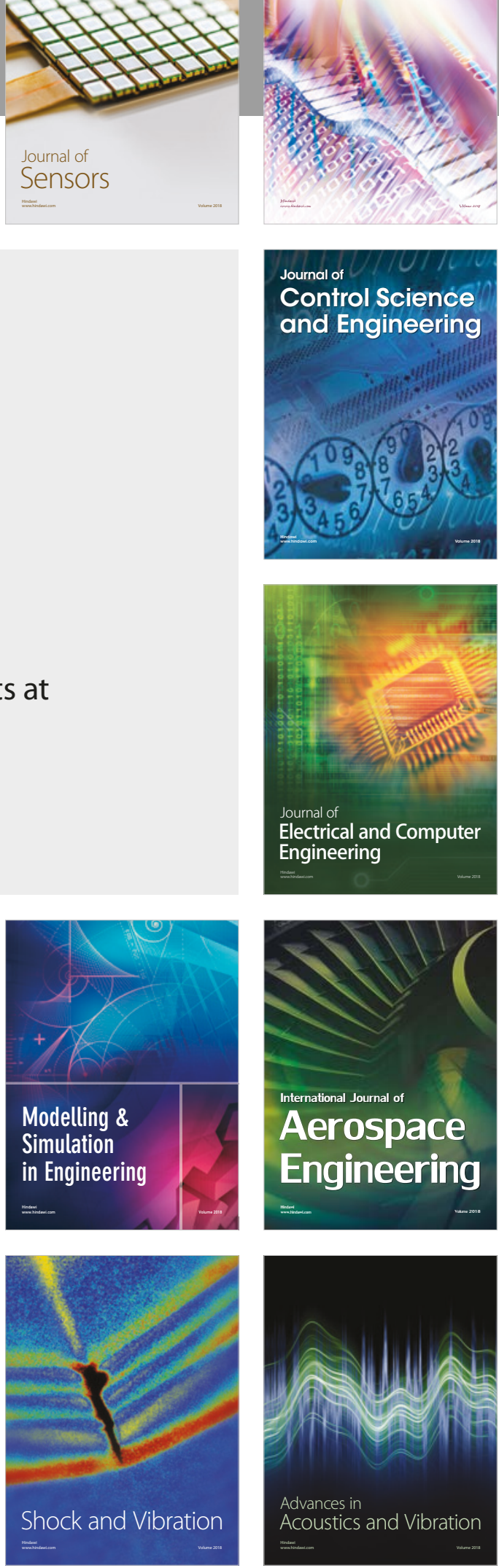